\title{
Presepsin levels in cirrhotic patients with bacterial infections and/or portal hypertension-related bleeding, presenting with or without acute kidney injury
}

\author{
Ioannis Elefsiniotis, Stefanos A. Tsakiris, Georgia Barla, Athanasia Tasovasili, Dimitrios Vrachatis, \\ Christos Mavrogiannis \\ "Agioi Anargyroi" General and Oncology Hospital of Kifisia, National and Kapodistrian University of Athens, Greece
}

Abstract

Academic Department of Internal Medicine, Hepatogastroenterology Unit, "Agioi Anargyroi" General and Oncology Hospital of Kifisia, National and Kapodistrian University of Athens, Greece, The study was partially supported by an unrestricted research grant from AbbVie Pharmaceuticals S.A. The authors state that AbbVie Pharmaceuticals S.A. had no involvement in the study design, collection, analysis, interpretation of data, writing of the report or the decision to submit the manuscript for publication.

Conflict of Interest: None

Correspondence to: Ioannis Elefsiniotis, Assoc. Professor of Medicine, Academic Department of Internal Medicine, "Agioi Anargyroi” General and Oncology Hospital of Kifisia, National and Kapodistrian University of Athens, Greece, Timiou Stavrou and Noufaron 14, Kalyftaki, N. Kifisia, 14564 Athens, Greece, e-mail: ielefs@nurs.uoa.gr

Received 28 March 2018; accepted 25 June 2018; published online 18 July 2018

DOI: https://doi.org/10.20524/aog.2018.0292

\section{Introduction}

The understanding, diagnosis and treatment of bacterial infections (BI) in cirrhotic patients continue to pose a challenge. Cirrhotic patients carry an increased risk of developing BI, sepsis, severe sepsis and septic shock, while infections at admission or during hospitalization occur in 25$35 \%$ of them [1]. The short-term mortality of cirrhotic patients with BI is extremely high, reaching $30 \%$ at one month and $63 \%$ during the first year [1]. Patients with liver cirrhosis are twice more likely to die from sepsis than patients without preexisting liver disease, and septic shock in this population is associated with a hospital mortality that may surpass $70 \%$ [2].

The development of biomarkers for the early diagnosis of BI in these patients is of major importance. The limitations of clinical examination, systemic inflammatory response 
syndrome (SIRS) criteria and currently used biomarkers, such as C-reactive protein (CRP) and procalcitonin (PCT), in patients with liver cirrhosis have raised awareness of this need [1]. The recognition of pathogen-associated molecular patterns, such as lipopolysaccharide (LPS), by membrane Toll-like receptors (TLR) and co-receptors activates effector cells of the innate immune system, including the monocyte-macrophage lineage, and provides an immediate response to microbial invasion [3]. One of these co-receptors is CD14, a glycoprotein expressed on monocytes and macrophages, which participates in the presentation of LPS to TLR [3,4]. Following exposure to LPS, expression of the membrane-bound form of CD14 (mCD14) decreases as a result of a complex process of proteolytic cleavage and internalization, which generates soluble forms of CD14 (sCD14) directly secreted by hepatocytes [3]. The N-terminal fragment of $13 \mathrm{kDa}$ has been identified as soluble CD14 subtype (sCD14-ST) or presepsin [5-7]. In 2005, sCD14-ST was described as highly specific in discriminating septic patients by conventional enzyme-linked immunosorbent assay (ELISA), but in 2011 a onestep chemiluminescent ELISA assay was validated as a simple and rapid bedside method with satisfactory results $[6,7]$.

Presepsin, a direct witness of activated monocytesmacrophages in response to pathogens, is able to signal an earlier increase compared with CRP and PCT, as was shown in experimental sepsis and burns studies, and demonstrates a unique capacity to distinguish the severity of sepsis in critically ill patients [3,4]. A cutoff value of $415 \mathrm{pg} / \mathrm{mL}$ is associated with $80 \%$ sensitivity and $81 \%$ specificity for a diagnosis of sepsis. In some studies, a comparison of areas under the receiver operating characteristic (ROC) curve (AUC) indicated that presepsin had a better diagnostic performance than CRP and PCT $[8,9]$. According to a recent multicenter study, compared with presepsin or PCT alone, presepsin in association with PCT had higher diagnostic accuracy for discriminating SIRS from sepsis [10].

Regarding acute or chronic liver diseases, more information is available concerning CD14 and sCD14 levels [11], rather than plasma presepsinlevels. The aim of our study was to evaluateplasma presepsin levels in a group of cirrhotic patients with compensated or decompensated liver disease, without documented BI or other portal hypertension-related complications and/or hepatocellular carcinoma (HCC) and to identify potential correlations with the severity of liver disease, using well defined scores for patients with liver cirrhosis, such as Child-Pugh and the model for end-stage liver disease (MELD) score. In addition, we evaluated presepsin levels in a subgroup of patients who presented with documented $\mathrm{BI}$ and/or portal hypertension-related bleeding (PHRB) with or without acute kidney injury (AKI).

\section{Patients and methods}

\section{Patients}

We prospectively evaluated the presepsin levels of consecutive cirrhotic patients without documented BI who presented in the outpatient Hepatology Unit of the "Agioi
Anargyroi" General and Oncology Hospital of Kifisia. Patient enrolment commenced in January 2015 and ended in March 2016, with an additional follow-up period of 6 months until September 2016. All patients had history of chronic liver disease and a diagnosis of compensated (confirmed by either liver biopsy or transient elastography) or decompensated liver cirrhosis. Contrast-enhanced computed tomography and magnetic resonance imaging, within the last 6 months prior to the baseline visit, were available in all the included patients so that patients with HCC could be excluded. Decompensated liver cirrhosis was defined by a history of at least one major cirrhosis-related complication, such as PHRB, hepatic encephalopathy, ascites with serum ascites albumin gradient $>1.1 \mathrm{~g} / \mathrm{dL}$, or jaundice. BI was ruled out by clinical examination, the absence of findings on chest X-ray suggestive of lower respiratory tract infections, and negative blood, urine and ascitic fluid cultures. The neutrophil count in ascitic fluid $\left(<250 / \mathrm{mm}^{3}\right)$ was also evaluated to exclude spontaneous bacterial peritonitis in cirrhotic patients with ascites. The investigations for ruling out BI were performed at baseline in all patients enrolled in the study and were repeated in the subgroup of patients who were reevaluated because of a complicating event. For the baseline characteristics of the study population, demographic data were collected through medical history, while hematological and biochemical tests were performed using standard laboratory methods. For the assessment of severity of chronic liver disease, well defined scores for cirrhotic patients, such as Child-Pugh and MELD, were used. The Child-Pugh score was calculated by adding the scores for each parameter-encephalopathy (none, 1 point: mild to moderate, 2 points; severe, 3 points), ascites (none, 1 point; mild to moderate, 2 points; severe, 3 points), bilirubin (<2 mg/dL, 1 point; $2-3 \mathrm{mg} / \mathrm{dL}, 2$ points; $>3 \mathrm{mg} / \mathrm{dL}, 3$ points), albumin ( $>3.5 \mathrm{~g} / \mathrm{dL}, 1$ point; $2.8-3.5 \mathrm{~g} / \mathrm{dL}, 2$ points; $<2.8 \mathrm{~g} / \mathrm{dL}$, 3 points), international normalized ratio (INR) $(<1.7,1$ point; 1.7-2.3, 2 points; $>2.3,3$ points) —and classifying chronic liver disease into Child-Pugh classes A to C (5-6 points, class A; 7-9 points, class B; 10-15 points, class C) [12]. The MELD score was calculated using the appropriate formula: MELD $=10 \times$ $[0.957 \times \operatorname{loge}($ creatinine $\mathrm{mg} / \mathrm{dL})+0.378 \times \log e($ bilirubin $\mathrm{mg} / \mathrm{dL})$ $+1.120 \times \operatorname{loge}($ INR $)+0.6431][13]$.

Written informed consent was collected from all the patients who participated in the current study. The study protocol was in accordance with the Declaration of Helsinki and was evaluated and approved by the Ethics Committee of the School of Health Sciences of the National and Kapodistrian University of Athens, Greece.

\section{Collection of samples and measurements}

The collection of samples was performed when the patients presented in the outpatient Hepatology Unit; demographic data were recorded and routine laboratory tests were ordered at the same time. Blood was collected in EDTA tubes from patients' peripheral veins and was analyzed promptly. Presepsin levels were assessed by PATHFAST (Mitsubishi Chemical Medience Corporation, Tokyo, Japan), a compact benchtop 
chemiluminescent enzyme immunoassay analyzer, which has been validated as a presepsin assay system for routine, fully automated, point-of-care measurements in plasma and whole blood samples, reducing the analysis time to $17 \mathrm{~min}[8,14]$.

\section{Subgroup of patients with event}

During the study and the additional follow-up period (6 months) a subset of patients suffered from an event, defined as documented BI with or without concomitant PHRB, or variceal bleeding without documented BI. These patients were admitted, hospitalized and reevaluated with whole blood collection and presepsin levels measurement at admission. BI was documented using the aforementioned criteria and PHRB was ruled out or confirmed clinically and by upper gastrointestinal endoscopy. A subgroup of these patients developed AKI according to the definition of the International Club of Ascites [15].

\section{Statistical analysis}

Differences between groups were evaluated by MannWhitney $U$ test for independent samples and by Wilcoxon signed-rank test for related samples. Spearman's rank correlation coefficient analysis was used to assess the association between two continuous variables. For prediction of mortality during the study and follow-up period, binary logistic regression analysis was performed. ROC curves were constructed for the estimation of the discriminative or predictive value of presepsin levels regarding several outcomes. The AUC was calculated for each studied outcome and coordinates of the ROC curve were used for the selection of cutoff values. Kaplan-Meier estimates were used to perform survival analysis and plot survival curves for patients categorized into groups according to their baseline presepsin levels compared with a specific cutoff value. In all cases a P-value $<0.05$ was considered statistically significant. Statistical analysis was performed using IBM SPSS Statistics for Macintosh, Version 23.

\section{Results}

\section{Patient characteristics}

A total of 108 patients with liver cirrhosis were included in the study. Detailed patient characteristics are shown in Table 1.

\section{Baseline presepsin levels in cirrhotic patients}

The mean baseline presepsin level of the entire study population ( $\mathrm{n}=108$ ) was mean (standard deviation [SD]) 440.4 (388.4) $\mathrm{pg} / \mathrm{mL}$ with a median (interquartile range [IQR]) of 335.0 (273.8) pg/mL. Patients with decompensated cirrhosis $(\mathrm{n}=53)$ exhibited significantly higher baseline presepsin levels_mean (SD) 599.1 (492.2) pg/mL; median (IQR) 441.0 (422.5) $\mathrm{pg} / \mathrm{mL}$-compared to patients with compensated cirrhosis ( $\mathrm{n}=55)$ - mean (SD) 287.5 (130.5) pg/mL; median (IQR) 262.0 (179.0) pg/mL $(\mathrm{P}<0.001)$ (Fig. 1A).

Table 1 Clinical and demographic patient characteristics. Values are presented as mean (SD) except for baseline presepsin levels, presented as $\underline{\text { median }(\mathrm{IQR})}$

\begin{tabular}{|c|c|c|c|c|c|c|c|}
\hline Characteristics & Overall & CLD & DLD & ALD/NAFLD & $\mathrm{HBV}$ & $\mathrm{HCV}$ & AI \\
\hline $\mathrm{n}$ & 108 & 55 & 53 & 34 & 15 & 44 & 15 \\
\hline Sex, $m / f$ & $78 / 30$ & $37 / 18$ & $41 / 12$ & $31 / 3$ & $13 / 2$ & $32 / 12$ & $2 / 13$ \\
\hline Age, years & $62.0(10.6)$ & $60.0(10.8)$ & $64.1(10.1)$ & $62.0(10.3)$ & $61.3(11.7)$ & $59.5(9.0)$ & $70.4(11.2)$ \\
\hline $\mathrm{WBC}, / \mu \mathrm{L}$ & $6554(2873)$ & $6577(2736)$ & $6531(3034)$ & $6979(2686)$ & $6909(4348)$ & $6349(2098)$ & $5839(3520)$ \\
\hline PLT, $\times 10^{3} / \mu \mathrm{L}$ & $151(92)$ & $163(78)$ & $138(103)$ & $158(115)$ & $138(89)$ & $158(73)$ & $125(72)$ \\
\hline INR & $1.26(0.45)$ & $1.07(0.12)$ & $1.45(0.57)$ & $1.29(0.26)$ & $1.45(0.79)$ & $1.19(0.47)$ & $1.18(0.18)$ \\
\hline Creatinine, $\mathrm{g} / \mathrm{dL}$ & $1.0(0.5)$ & $0.9(0.2)$ & $1.1(0.7)$ & $0.9(0.2)$ & $1.2(1.3)$ & $0.9(0.2)$ & $1.0(0.3)$ \\
\hline Sodium, $\mathrm{mmol} / \mathrm{L}$ & $139.4(3.8)$ & $140.8(2.7)$ & $138.0(4.3)$ & $138.9(3.8)$ & $138.7(4.1)$ & $140.1(3.1)$ & $139.1(5.3)$ \\
\hline Bilirubin, g/dL & $2.0(3.1)$ & $0.9(0.9)$ & $3.1(4.0)$ & $2.6(3.7)$ & $2.2(2.6)$ & $1.6(3.2)$ & $1.6(1.3)$ \\
\hline Albumin, g/dL & $3.7(0.6)$ & $4.1(0.4)$ & $3.4(0.6)$ & $3.6(0.6)$ & $3.6(0.6)$ & $3.9(0.5)$ & $3.7(0.5)$ \\
\hline MELD score & $10.6(5.1)$ & $7.7(1.7)$ & $13.6(5.6)$ & $11.7(4.3)$ & $12.7(7.7)$ & $9.2(4.6)$ & $10.5(3.8)$ \\
\hline Child-Pugh score & $6.3(1.8)$ & $5.1(0.5)$ & $7.5(1.9)$ & $6.6(1.8)$ & $6.8(1.9)$ & $5.8(1.6)$ & $6.5(1.9)$ \\
\hline $\begin{array}{l}\text { Baseline presepsin, } \\
\mathrm{pg} / \mathrm{mL}\end{array}$ & $335.0(273.8)$ & $262.0(179.0)$ & $441.0(442.5)$ & $356.5(320.0)$ & $332.0(365.0)$ & $306.0(198.3)$ & $496.0(757.0)$ \\
\hline
\end{tabular}




\section{Baseline presepsin levels are correlated with the severity of chronic liver disease}

Significantly higher baseline presepsin levels were observed in the Child-Pugh B/C group ( $\mathrm{n}=37$ )-mean (SD) 674.4 (500.4) pg/mL; median (IQR) 566.0 (356.0) pg/mL-compared with the Child-Pugh A group ( $\mathrm{n}=71)$-mean (SD) 318.5 (240.4) pg/mL; median (IQR) 263.0 (190.0) pg/mL $(\mathrm{P}<0.001)$ (Fig. 1B). Additionally, the baseline presepsin levels of cirrhotic patients with MELD score $\geq 15$ ( $n=18$ ) — mean (SD) 620.3 (417.5) pg/mL; median (IQR) 442.5 (365.0) pg/mL-were significantly higher than the corresponding ones of patients with MELD score $<15$ ( $\mathrm{n}=90$ )—mean (SD) 404.4 (374.5) pg/mL; median (IQR) 314.5 (243.8) pg/mL $(\mathrm{P}<0.001)$ (Fig. 1C). A MELD score of 15 was selected as a cutoff to differentiate patients with advanced chronic liver disease, since it has also been used for selecting cirrhotic patients for pre-transplant evaluation [16].

\section{Presepsin levels in cirrhotic patients admitted with event}

During the study period and follow up a subgroup of patients $(18.5 \%, 20 / 108)$ presented with a complicating event (documented BI and/or variceal bleeding) with or without a concomitant portal hypertension-related complication (hepatic encephalopathy, AKI). Almost all patients in this subgroup (19/20) had decompensated liver disease at baseline evaluation because of at least one previous decompensating event (17/19 with ascites, $1 / 19$ with recurrent episodes of hepatic encephalopathy and $1 / 19$ with jaundice) and only $1 / 20$ did not have any history of a decompensating event in the past. These patients were hospitalized and reevaluated at admission (Table 2). BI was documented in 18 patients $(18 / 20)$, of whom $6(6 / 18)$ suffered from concomitant PHRB (2/6 patients with PHRB related to portal hypertensive gastropathy and $4 / 6$ patients with variceal bleeding), whereas 2 patients $(2 / 20)$ were admitted because of variceal bleeding without documented BI. Nine patients were admitted with documented bacteremia (5 of them with concomitant documented spontaneous bacterial peritonitis), 5 with a culture-positive urinary tract infection and 4 with a documented lower respiratory tract infection. Presepsin levels of the admitted cirrhotic patients ( $\mathrm{n}=20$ )-mean (SD) 1438.0 (1247.2) pg/mL, median (IQR) 943.0 (1593.0) $\mathrm{pg} / \mathrm{mL}$ - were remarkably higher compared to their baseline levels—mean (SD) 725.3 (602.8) pg/mL, median (IQR) 528.0 (480.0) pg/mL $(\mathrm{P}<0.001)$.



Figure 1 Graph of presepsin levels according to severity of chronic liver disease. (A) Patients with decompensated liver cirrhosis ( $\mathrm{n}=53$ ) exhibited significantly higher baseline presepsin levels, $P<0.001$. (B) Patients in the Child-Pugh $B / C$ group $(n=37)$ exhibited significantly higher baseline presepsin levels, $P<0.001$. (C) The baseline presepsin levels of patients with model for end-stage liver disease (MELD) score $\geq 15$ ( $n=18$ ) were significantly higher than those of patients with MELD score $<15(\mathrm{n}=90), \mathrm{P}<0.001$. (D) The patients in the acute kidney injury (AKI) group ( $\mathrm{n}=10$ ) exhibited significantly higher presepsin levels, at both baseline and admission compared to the patients in the non-AKI group ( $\mathrm{n}=10)$, $\mathrm{P}<0.05$. The Mann-Whitney $U$ test for independent samples was used to assess differences between groups. The graphs and error bars depict median values and $95 \%$ CI for median, respectively 
Baseline presepsin levels of the patients who presented with a complicating event $(n=20)$, significantly correlated with the corresponding levels at admission (Spearman's rho statistically significant at the 0.01 level) (Fig. 2).

\section{Patients who developed AKI exhibited higher presepsin levels both at baseline and at hospital admission}

Ten of the 20 hospitalized patients (50\%) developed AKI and all of them had a fatal outcome. The patients in the AKI group $(n=10)$ exhibited significantly higher presepsin levels, both at baseline-mean (SD) 936.3 (730.1) pg/ $\mathrm{mL}$; median (IQR) 714.0 (637.0) pg/mL-and at hospital admission-mean (SD) 1827.3 (1118.8) $\mathrm{pg} / \mathrm{mL}$; median (IQR) 1616.0 (1470.0) pg/mL-compared with the cirrhotic patients in the non-AKI group ( $\mathrm{n}=10)$-baseline mean (SD) 514.3 (367.7) pg/mL; median (IQR) 404.0 (419.0) pg/mL and admission mean (SD) 1048.7 (1302.1) pg/mL; median (IQR) 616.5 (664.0) pg/mL $(\mathrm{P}<0.05)$ (Fig. 1D). However, the percentage change in presepsin levels from baseline to admission did not differ significantly between the AKI and the non-AKI group (data not shown).

\section{Baseline presepsin levels predicts 1-year and 3-month mortality}

During the follow-up period, 20 cirrhotic patients died (10 of them with MELD score $\geq 15,2$ with compensated liver disease): in 17 patients death was due to their liver disease, while in the remaining 3 (including 2 with compensated liver disease) it was from causes unrelated to their liver disease (2 died from major cardiovascular events and one from hemorrhagic stroke). Twelve deaths were observed within the first 3 months of the observational period and 10 of them were liver-related.

Binary logistic regression analysis was performed to investigate how 1-year and 3-month mortality can be predicted by baseline presepsin levels. Taking into account patients' age, sex, liver cirrhosis etiology, Child-Pugh score ( $\geq 7$ or $<7$ points), MELD score ( $\geq 15$ or $<15$ points) and baseline presepsin levels $(\geq 600$ or $<600 \mathrm{pg} / \mathrm{mL}$ ), only MELD score $\geq 15$ points $-\mathrm{B}$ (standard error [SE]) $1.76(0.90), \mathrm{P}=0.05$, odds ratio $[\mathrm{OR}]$ (95\%CI for OR) 5.80 (1.00-35.66) - and baseline presepsin levels $\geq 600 \mathrm{pg} / \mathrm{mL}-\mathrm{B}$ (SE) 1.77 (0.82), $\mathrm{P}<0.05$, OR (95\%CI for OR) 5.88 (1.18-29.27)-were retained in the model for death within the first year (1-year mortality). In the models for early death (3-month mortality), liver disease-related death within the first year (liver disease-related 1-year mortality) and liver disease-related early death (liver disease-related 3-month mortality), only baseline presepsin $\geq 600 \mathrm{pg} / \mathrm{mL}$ was retained, with B (SE) 1.75 (0.83), P<0.05, OR (95\%CI for OR) 5.78 (1.1329.56), B (SE) 2.22 (0.92), $\mathrm{P}<0.05$, OR (95\%CI for OR) 9.22 (1.53-55.63), and B (SE) 2.58 (1.07), $\mathrm{P}<0.05$, OR (95\%CI for OR) 13.22 (1.61-108.42), respectively.

ROC curves were constructed and values of AUC were calculated to estimate the discriminative or predictive value of baseline presepsin levels regarding the aforementioned outcomes. The calculated AUC values of baseline presepsin levels were: for 1-year mortality AUC (95\%CI) 0.840 (0.7520.929 ), asymptotic significance $\mathrm{P}<0.001$; for 3 -month mortality AUC (95\%CI) 0.826 (0.715-0.937), asymptotic significance $\mathrm{P}<0.001$; for liver disease-related 1-year mortality AUC (95\%CI) 0.832 (0.731-0.933), asymptotic significance $\mathrm{P}<0.001$; and for liver disease-related 3-month mortality AUC (95\%CI) 0.825 (0.684-0.967), asymptotic significance $\mathrm{P}<0.01$.

At a cutoff of $436.5 \mathrm{pg} / \mathrm{mL}$, baseline presepsin levels exhibited $80 \%$ sensitivity and $79.5 \%$ specificity for the prediction of 1-year mortality (Fig. 3), 83.3\% sensitivity and

Table 2 Summary of baseline and admission presepsin levels in subgroups of patients. The values of presepsin levels are presented as median (IQR) and lower bound 95\%CI - upper bound 95\%CI for median

\begin{tabular}{lccc}
\hline Presepsin levels in patient subgroups & $\mathrm{n}$ & Median (IQR), pg/mL & $95 \% \mathrm{CI}$ for median, pg/mL \\
\hline Total, baseline & 108 & $335.0(273.8)$ & $289.0-393.0$ \\
Compensated, baseline & 55 & $262.0(179.0)$ & $226.0-327.0$ \\
Decompensated, baseline & 53 & $441.0(422.5)$ & $386.0-600.0$ \\
Child-Pugh A, baseline & 71 & $263.0(190.0)$ & $226.5-317.5$ \\
Child-Pugh B/C, baseline & 37 & $566.0(356.0)$ & $441.0-687.0$ \\
MELD<15, baseline & 90 & $314.5(243.8)$ & $263.0-369.0$ \\
MELD $\geq 15$, baseline & 18 & $442.5(365.0)$ & $404.0-667.0$ \\
Event group, baseline & 20 & $528.0(480.0)$ & $404.0-811.4$ \\
Event group, at admission & 20 & $943.0(1593.0)$ & $621.0-1616.0$ \\
non-AKI group, baseline & 10 & $404.0(419.0)$ & $270.0-654.0$ \\
non-AKI group, at admission & 10 & $616.5(664.0)$ & $438.0-995.0$ \\
AKI group, baseline & 10 & $714.0(637.0)$ & $441.0-1021.0$ \\
\hline AKI group, at admission & 10 & $1616.0(1470.0)$ & $891.0-2276.0$ \\
\hline
\end{tabular}

AKI, acute kidney injury; IQR, interquartile range; 95\%CI, 95\% confidence interval 
$75.0 \%$ specificity for the prediction of 3-month mortality, and $76.5 \%$ sensitivity and $76.9 \%$ specificity for the prediction of liver disease-related 1-year mortality, whereas baseline

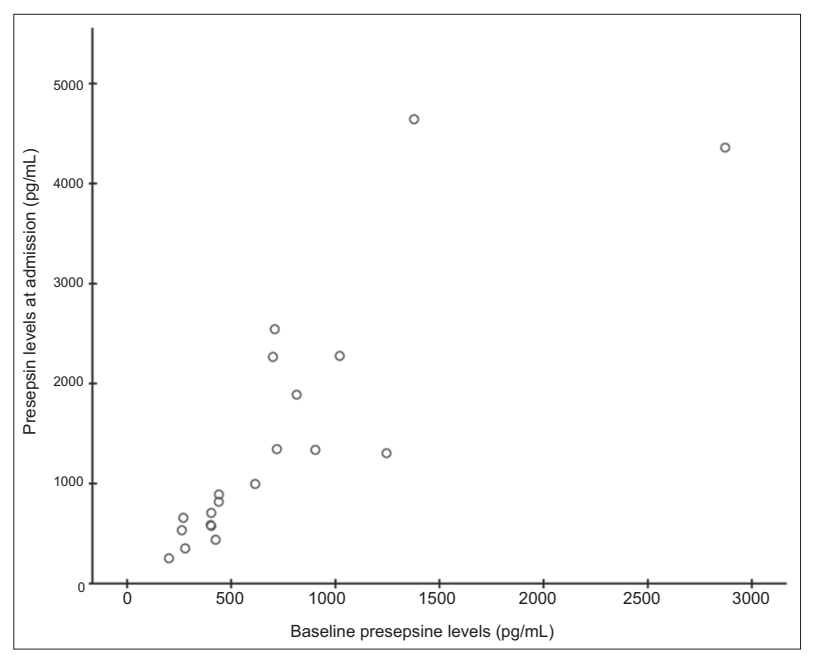

Figure 2 Graph of correlation between baseline presepsin levels of patients with event and presepsin levels at admission. Presepsin levels at admission for patients with an event $(n=20)$ were significantly higher than their baseline levels, $\mathrm{P}<0.001$. The Wilcoxon signed-rank test for related samples was used to compare differences between groups and Spearman's rank correlation coefficient analysis was used to assess the association between two variables. The baseline levels of presepsin correlated with the levels at admission, with the correlation being significant at the 0.01 level

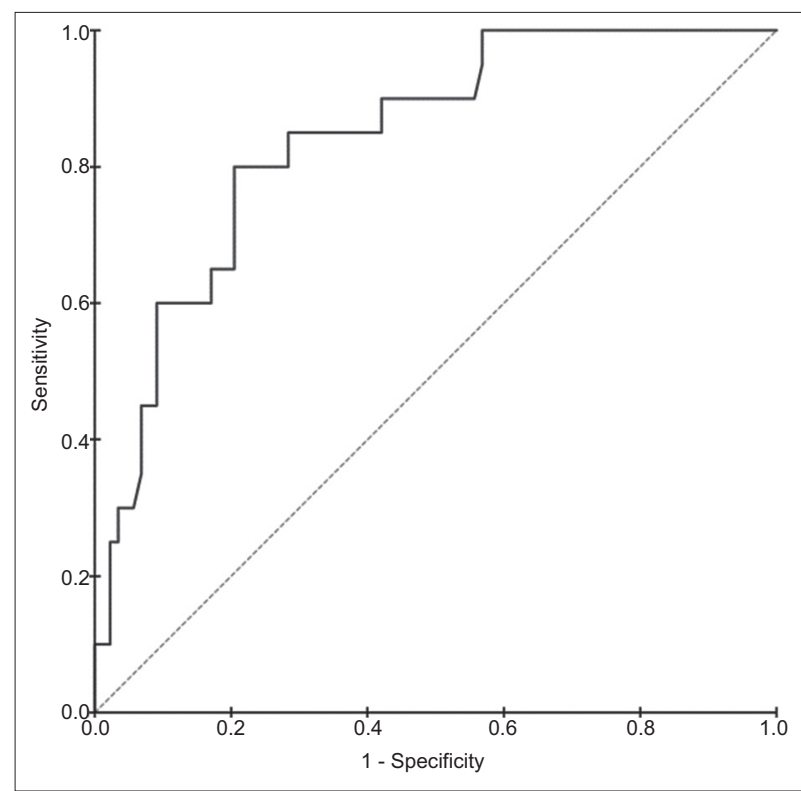

Figure 3 Receiver operating characteristic (ROC) curve of baseline presepsin levels for 1-year mortality. The area under the ROC curve (AUC) was calculated to estimate the predictive value of baseline presepsin levels for 1-year mortality, AUC ( $95 \%$ confidence interval): $0.840(0.752-0.929)$, asymptotic significance $\mathrm{P}<0.001$. At a cutoff of $436.5 \mathrm{pg} / \mathrm{mL}$, baseline presepsin levels exhibited sensitivity $80 \%$ and specificity $79.5 \%$ for prediction of 1 -year mortality presepsin levels at a higher cutoff $(607.5 \mathrm{pg} / \mathrm{mL})$ exhibited $77.8 \%$ sensitivity and $86.9 \%$ specificity for the prediction of liver disease-related 3-month mortality.

The Kaplan-Meier method was applied to estimate and plot the survival curves for the patients of the study population, when they were categorized into two groups based on their baseline presepsin levels being higher/equal or lower than the cutoff value of $600 \mathrm{pg} / \mathrm{mL}$. For the time variable, the period from the date of baseline presepsin level assessment to the date of last follow up was used and a log rank test was performed to compare the survival curves statistically, giving $\mathrm{P}<0.001$, which indicates a highly significant difference between the survival curves of the two groups (Fig. 4).

\section{Discussion}

Presepsin has recently been proposed as a valuable biomarker for the diagnosis and prognosis of sepsis. However, there are few data in the literature concerning the usefulness of plasma presepsin levels for the diagnosis of sepsis in patients with liver cirrhosis. In the current study we found that baseline presepsin levels in uncomplicated cirrhotic patients were elevated compared with the corresponding levels observed in the general population. According to one published study, the reference presepsin concentrations in individuals aged 18-72

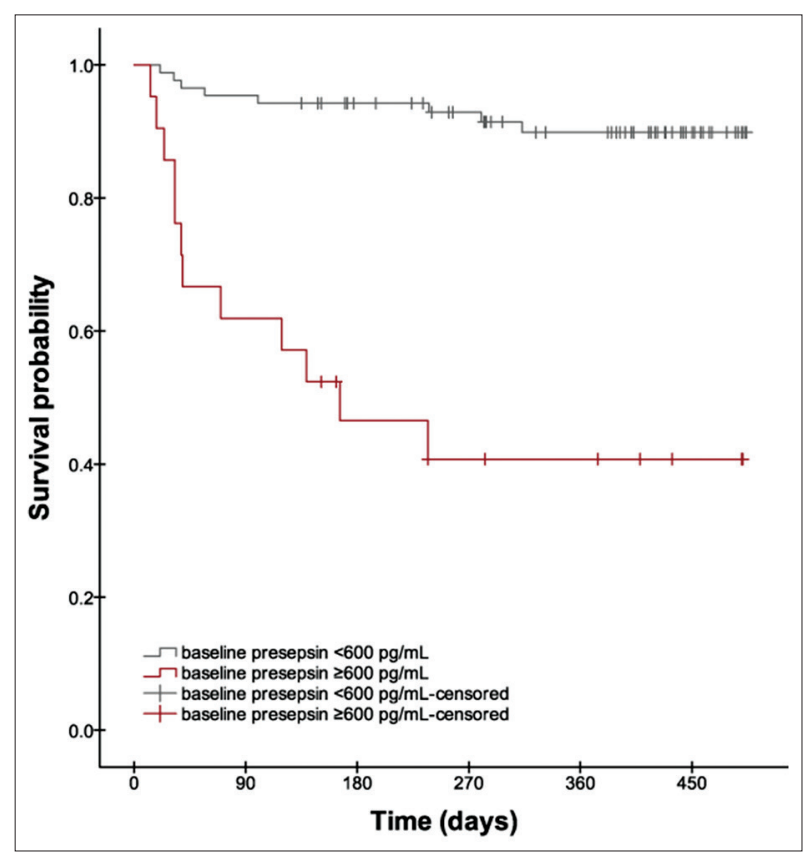

Figure 4 Kaplan-Meier survival curves of cirrhotic patients stratified by their baseline presepsin levels. The patients of the study population were categorized into two groups based on their baseline presepsin levels being higher/equal $(n=21)$ or lower $(n=87)$ than the cutoff value of $600 \mathrm{pg} / \mathrm{mL}$, the Kaplan-Meier method was applied to plot the corresponding survival curves and a log rank test was performed to compare them statistically. There was a highly significant difference between the survival curves of the two groups $(\mathrm{P}<0.001)$ 
years, without inflammatory disease or BI, were 55-184 pg/mL and were not particularly influenced by age [17], whereas in the cirrhotic population of our study the mean baseline presepsin level was $440.4 \mathrm{pg} / \mathrm{mL}$, with a median of $335.0 \mathrm{pg} / \mathrm{mL}$. It has been proposed that, even in the early stages of cirrhosis, minor and persistent paracellular translocation of microbial products initiates an enhanced proinflammatory cytokine response aimed at enhancing bacterial defense [18]. As a direct witness of activated monocytes/macrophages, sCD14-and therefore soluble sCD14 subtype (presepsin)_could be perceived as a good candidate biomarker of the host immune response to circulating LPS, reflective of the persistent bacterial translocation and/or hidden BI of cirrhotic patients $[3,11]$. This concept could account for the elevated presepsin levels observed in cirrhotic patients without documented BI. It is important to note that $35.2 \%(38 / 108)$ of cirrhotic patients without documented BI ( 30 with decompensated and 8 with compensated liver disease) in our study population exhibited mean baseline presepsin levels higher than the cutoff of 415 $\mathrm{pg} / \mathrm{mL}$ proposed for the diagnosis of sepsis in critically ill patients [8].

Baseline presepsin levels were significantly higher in patients with decompensated cirrhosis than in those with compensated cirrhosis. It is well known that the rate and degree of translocating microbial products increase continuously from the early stages of liver cirrhosis and portal hypertension to the advanced stages where there is also translocation of viable bacteria to the mesenteric lymph nodes (MLN) $[18,19]$. The higher baseline presepsin levels in patients with decompensated liver disease without established BI could reflect the increased rate and degree of bacterial translocation in those patients compared to cirrhotics with compensated liver disease [19]. LPS-binding protein, considered as a biomarker of gramnegative bacterial translocation, is significantly increased in cirrhotic patients, while intestinal permeability is higher in cirrhotic patients with ascites, but not in those without, compared to healthy controls [19]. Severe liver disease, as expressed by the well-defined Child-Pugh and MELD scores is also associated with an increased rate and degree of bacterial translocation [18]. Systemic endotoxin, another biomarker of pathological bacterial translocation, is increased in relation to the severity of chronic liver disease graded by the Child-Pugh classification system and Child $\mathrm{C}$ cirrhotic patients exhibit a higher rate of culturable bacterial translocation to MLN compared with Child A/B patients [18]. In accordance with this concept, baseline presepsin levels in our study population were significantly higher in patients with Child-Pugh class B/C than in the Child-Pugh A group, and in patients with MELD score $\geq 15$ compared with the MELD $<15$ group. It seems that the evaluation of plasma or whole blood presepsin levels in uncomplicated cirrhotic patients, using the fully automated, bedside and rapid PATHFAST assay, could indirectly reflect the level of bacterial translocation and could roughly outline the severity of portal hypertension and the patients' prognosis.

The usefulness of presepsin in cirrhotic patients during hospitalization for a documented BI and/or variceal bleeding was evaluated in a subgroup of patients admitted with such an event during the follow-up period. The presepsin levels at admission were significantly higher than the corresponding baseline levels for the admitted cirrhotic patients and correlated with them. These results are in accordance with data indicating that presepsin is a useful biomarker of BI, increasing significantly in the early stages of sepsis [3]. These findings suggest that plasma presepsin levels could be a useful biomarker for the diagnosis of BI in cirrhotic patients, but the cutoff values need further evaluation and should be different from those used in other groups of patients. In the two cases of variceal bleeding without concomitant documented BI, a significant increase in presepsin levels from baseline was also expected, since bleeding in cirrhotic patients frequently leads to enhanced bacterial translocation and results in documented BI in a considerable proportion of them [19]. Moreover, misdiagnosed BI in cirrhotic patients with conditions such as transient bacteremia has been proposed as a possible triggering for the aggravation of preexisting portal hypertension, ultimately resulting in PHRB [19]. Preexisting increased intestinal permeability in uncomplicated cirrhotic patients with portal hypertension has been proposed as the underlying mechanism [18] and could account for the correlation of baseline presepsin levels with levels at admission during BI and/or PHRB.

Half of the cirrhotic patients admitted with an event developed AKI and exhibited significantly higher presepsin levels, both at baseline and at hospital admission, compared with the admitted patients who did not develop AKI. Renal function is frequently impaired in cirrhotic patients with decompensated liver disease and especially in those with higher MELD scores [20], an observation that could partially explain the positive correlation of presepsin levels with both Child-Pugh stage and MELD score in uncomplicated cirrhotic patients. Significantly elevated presepsin levels have also been described in patients with renal dysfunction without BI [21], a parameter that could contribute to the elevated levels observed in patients with uncomplicated decompensated liver disease, compared to patients with compensated disease, as well as in those infected and presenting with AKI. However, in our study population the percentage change in presepsin levels from baseline to admission did not differ significantly between the AKI and the non-AKI group-an indirect indication that higher presepsin production, rather than reduced renal excretion, in patients with $\mathrm{BI}$ and pre-existing portal hypertension and/ or bacterial translocation, mainly accounts for the elevated presepsin levels observed in those patients. This finding should be further explored in other studies evaluating cirrhotic patients, with the inclusion of a higher percentage of patients with renal impairment.

Baseline presepsin levels could predict 1-year and 3-month mortality, as well as 1-year liver disease-related mortality and 3-month liver disease-related mortality, as shown by the results of the logistic regression analysis, which included patients' age, sex, liver cirrhosis etiology, MELD score $\geq$ or $<15$, Child-Pugh score $\geq$ or $<7$, and baseline presepsin levels $\geq$ or $<600 \mathrm{pg} / \mathrm{mL}$. In the analysis for 1 -year mortality, only MELD score $\geq 15$ and baseline presepsin $\geq 600 \mathrm{pg} / \mathrm{mL}$ were retained, whereas in the analysis for 3-month mortality, 1-year liver diseaserelated mortality and 3-month liver disease-related mortality, 
only baseline presepsin $\geq 600 \mathrm{pg} / \mathrm{mL}$ remained statistically significant. In a recent study by Papp et al, presepsin levels in cirrhotic patients failed to detect any prognostic relevance with respect to 1-month mortality after adjusting for MELD score [22]. These results are not discrepant with the findings of our study, since MELD score was included in our binary logistic regression analysis models only as a categorical variable $(<$ or $\geq 15)$. Our results indicate that plasma presepsin levels of uncomplicated cirrhotic patients without documented BI or variceal bleeding, especially those with advanced liver disease (i.e., those with decompensated liver disease, Child-Pugh stage $B$ or $C$ and/or MELD score $\geq 15$ ), could serve as a useful additional marker, along with MELD score, for discriminating cirrhotic patients at extremely high risk of an early unfavorable and frequently fatal outcome.

Our single-center study certainly has some limitations. The sample size of the cirrhotic patients was relatively small, especially with regard to the number of events recorded during the study and follow-up period. This necessitates the replication of the results in larger samples with long-term follow up and recording of events and outcomes. A better understanding of presepsin kinetics during BI in cirrhotic patients is needed, and the contribution of intestinal permeability, bacterial translocation and kidney function to presepsin levels remains to be further elucidated. The unmet challenges in the diagnosis of BI in cirrhotic patients also pose a limitation for our study, since false negative cultures could present a source of bias for ruling out $\mathrm{BI}$.

\section{Summary Box}

\section{What is already known:}

- Diagnosis of bacterial infections (BI) remains a challenge in cirrhotic patients

- The use of common biomarkers in cirrhosis has several limitations

- Presepsin has been proposed as a valuable sepsis biomarker

What the new findings are:

- Baseline presepsin levels are higher in cirrhotic patients with higher Child-Pugh (B/C vs. A) and model for end-stage liver disease (MELD) scores ( $\geq 15$ vs. $<15$ ), without clinically overt and/or laboratory confirmed BI

- BI and/or variceal bleeding further increase presepsin levels in cirrhotic patients

- Baseline presepsin levels could be evaluated as an additional biomarker, along with MELD score, in cirrhotic patients with advanced liver disease, for discriminating patients at an extremely high risk of an early complicating event with a frequently fatal outcome
In summary, plasma presepsin levels are elevated in uncomplicated cirrhotic patients, especially in those with decompensated liver disease and Child-Pugh stages B/C and/or high MELD scores. BI and/or variceal bleeding further increase plasma presepsin levels, especially in patients with advanced liver disease who develop AKI during their hospitalization. Finally, baseline presepsin levels, using the cutoff of $600 \mathrm{pg} / \mathrm{mL}$, could be used in addition to MELD score to predict 1-year and 3-month, all-cause and liver disease-related mortality, a finding that could play an important role in the prediction of shortterm outcomes in these severely ill patients. The usefulness of presepsin levels for the confirmation of BI in cirrhotic patients, as well as for the prediction of their clinical outcome, should be further evaluated.

\section{References}

1. Jalan R, Fernandez J, Wiest R, et al. Bacterial infections in cirrhosis: a position statement based on the EASL Special Conference 2013. J Hepatol 2014;60:1310-1324.

2. Plessier A, Denninger $\mathrm{MH}$, Consigny $\mathrm{Y}$, et al. Coagulation disorders in patients with cirrhosis and severe sepsis. Liver Int 2003;23:440-448.

3. Chenevier-Gobeaux C, Borderie D, Weiss N, Mallet-Coste T, Claessens YE. Presepsin (sCD14-ST), an innate immune response marker in sepsis. Clin Chim Acta 2015;450:97-103.

4. Wu J, Hu L, Zhang G, Wu F, He T. Accuracy of Presepsin in sepsis diagnosis: a systematic review and meta-analysis. PLoS One 2015;10:e133057.

5. Mussap M, Noto A, Fravega $M$, Fanos V. Soluble CD14 subtype presepsin (sCD14-ST) and lipopolysaccharide binding protein (LBP) in neonatal sepsis: new clinical and analytical perspectives for two old biomarkers. J Matern Fetal Neonatal Med 2011;24(Suppl 2):12-14.

6. Yaegashi Y, Shirakawa K, Sato N, et al. Evaluation of a newly identified soluble CD14 subtype as a marker for sepsis. J Infect Chemother 2005;11:234-238.

7. Shirakawa K, Naitou K, Hirose J, Takahashi T, Furusako S. Presepsin (sCD14-ST): development and evaluation of one-step ELISA with a new standard that is similar to the form of presepsin in septic patients. Clin Chem Lab Med 2011;49:937-939.

8. Shozushima T, Takahashi G, Matsumoto N, Kojika M, Okamura Y, Endo S. Usefulness of presepsin (sCD14-ST) measurements as a marker for the diagnosis and severity of sepsis that satisfied diagnostic criteria of systemic inflammatory response syndrome. J Infect Chemother 2011;17:764-769.

9. Ishikura H, Nishida T, Murai A, et al. New diagnostic strategy for sepsis-induced disseminated intravascular coagulation: a prospective single-center observational study. Crit Care 2014;18:R19.

10. Takahashi G, Shibata S, Ishikura H, et al. Presepsin in the prognosis of infectious diseases and diagnosis of infectious disseminated intravascular coagulation: a prospective, multicentre, observational study. Eur J Anaesthesiol 2015;32:199-206.

11. Sandler NG, Koh C, Roque A, et al. Host response to translocated microbial products predicts outcomes of patients with HBV or HCV infection. Gastroenterology 2011;141:1220-1230, 1230.e1-e3.

12. Pugh RN, Murray-Lyon IM, Dawson JL, Pietroni MC, Williams R. Transection of the oesophagus for bleeding oesophageal varices. Br J Surg 1973;60:646-649.

13. Wiesner R, Edwards E, Freeman R, et al; United Network for 
Organ Sharing Liver Disease Severity Score Committee. Model for end-stage liver disease (MELD) and allocation of donor livers. Gastroenterology 2003;124:91-96.

14. Okamura Y, Yokoi H. Development of a point-of-care assay system for measurement of presepsin (sCD14-ST). Clin Chim Acta 2011;412:2157-2161.

15. Angeli P, Ginès P, Wong F, et al. Diagnosis and management of acute kidney injury in patients with cirrhosis: revised consensus recommendations of the International Club of Ascites. J Hepatol 2015;62:968-974.

16. Elwir S, Lake J. Current status of liver allocation in the United States. Gastroenterol Hepatol (N Y) 2016;12:166-170.

17. Giavarina D, Carta M. Determination of reference interval for presepsin, an early marker for sepsis. Biochem Med (Zagreb) 2015;25:64-68.
18. Wiest R, Lawson M, Geuking M. Pathological bacterial translocation in liver cirrhosis. J Hepatol 2014;60:197-209.

19. Bernardi M, Moreau R, Angeli P, Schnabl B, Arroyo V. Mechanisms of decompensation and organ failure in cirrhosis: From peripheral arterial vasodilation to systemic inflammation hypothesis. J Hepatol 2015;63:1272-1284.

20. Francoz C, Glotz D, Moreau R, Durand F. The evaluation of renal function and disease in patients with cirrhosis. $J$ Hepatol 2010;52:605-613.

21. Nagata T, Yasuda Y, Ando M, et al. Clinical impact of kidney function on presepsin levels. PLoS One 2015;10:e0129159.

22. Papp M, Tornai T, Vitalis Z, et al. Presepsin teardown - pitfalls of biomarkers in the diagnosis and prognosis of bacterial infection in cirrhosis. World J Gastroenterol 2016;22:9172-9185. 